\title{
The effect of exosomes in transferring TET signaling alterations
}

\author{
SERGIU PASCA ${ }^{1, *}$; ANCUTA JURJ ${ }^{2}$ \\ 1 Department of Hematology, Iuliu Hatieganu University of Medicine and Pharmacy, Cluj-Napoca, 400012, Romania \\ 2 Research Center for Functional Genomics, Biomedicine and Translational Medicine, Iuliu Hatieganu University of Medicine and Pharmacy, Cluj-Napoca, \\ 400337, Romania
}

Key words: TET, Exosomes, Immunity, Intercellular communication

\begin{abstract}
Ten eleven translocation (TET) enzymes are composed of three representatives: TET1/2/3 which are involved in the hydroxymethylation of methylated cytosines. Because of the wide array of processes that are governed by these epigenetic marks, there have been a wide range of clinical effects associated with TET alterations. Even though many research groups have focused on analyzing the effect of TET alterations within certain cells, few have taken into consideration the effect of TET in the context of intercellular communication. One important entity through which intercellular communication occurs is represented by exosomes. Thus, in the current viewpoint we discussed the direct transfer of TET by exosomes, its alterations in the cell targeted by exosomes and the effect of TET alterations on exosome secretion.
\end{abstract}

\section{Introduction}

Ten eleven translocation (TET) enzymes are composed of three representatives: TET1/2/3 which are involved in the hydroxymethylation of methylated cytosines. This not only initiates the process of demethylation, but also determines the generation of another epigenetic mark, 5-hydroxymethylcytosine (Kohli and Zhang, 2013; Wu and Zhang, 2017). Because of the wide array of processes that are governed by these epigenetic marks, there have been a wide range of clinical effects associated with TET alterations. The most common fields in which these alterations have an impact are represented by the fields of oncology (Aslanyan et al., 2014) and immunity, including autoimmunity and atherosclerosis (Tanaka et al., 2020; Oh et al., 2020; Pasca et al., 2021). Even though many research groups have focused on analyzing the effect of TET alterations within certain cells, few have taken into consideration the effect of TET in the context of intercellular communication (Kohli and Zhang, 2013; Wu and Zhang, 2017). One important entity through which intercellular communication occurs is represented by exosomes. These are nanoscale vesicles that transfer a variety of molecular species between different cell types or between different cells of the same type (Jurj et al., 2020). Because of this variety, it is with no doubt possible for them

*Address correspondence to: Sergiu Pasca, pasca.sergiu123@gmail.com Received: 17 June 2021; Accepted: 02 September 2021 to also transfer TET enzymes or factors implicated in the alteration of TET in the target cell. Added to this, because of the multitude of effects that TET enzymes have, it is also possible for it to influence exosome loading and secretion.

Thus, we will further discuss the direct transfer of TET by exosomes, its alterations in target cells and the effect of TET alterations on exosome secretion (Fig. 1).

\section{TET direct transfer}

One manner through which exosomes can alter the target cell is through the direct transfer of proteins and increase in their level within the target cell. In this context, it has been shown that CD137 activation reduces the loading of TET2 within exosomes secreted by endothelial cells, thus reducing the TET2 that would normally be transferred to smooth muscle cells. Normally, the physiological transfer of TET2 from endothelial cells protects smooth muscle cells from phenotype switch, inducing an anti atherosclerotic effect. The reduction of TET2 transfer is associated with an increased phenotype switch in smooth muscle cells and atherosclerosis promotion (Li et al., 2020).

\section{TET alteration within the target cell}

Current studies have shown that TET activity in the target cell can be altered either through the influence of microRNAs or through certain metabolites shuttled via exosomes. Nonetheless, because of the small number of studies currently present in the literature, there might also be other manners through which TET enzymes within the target cell might be affected by exosomes. 


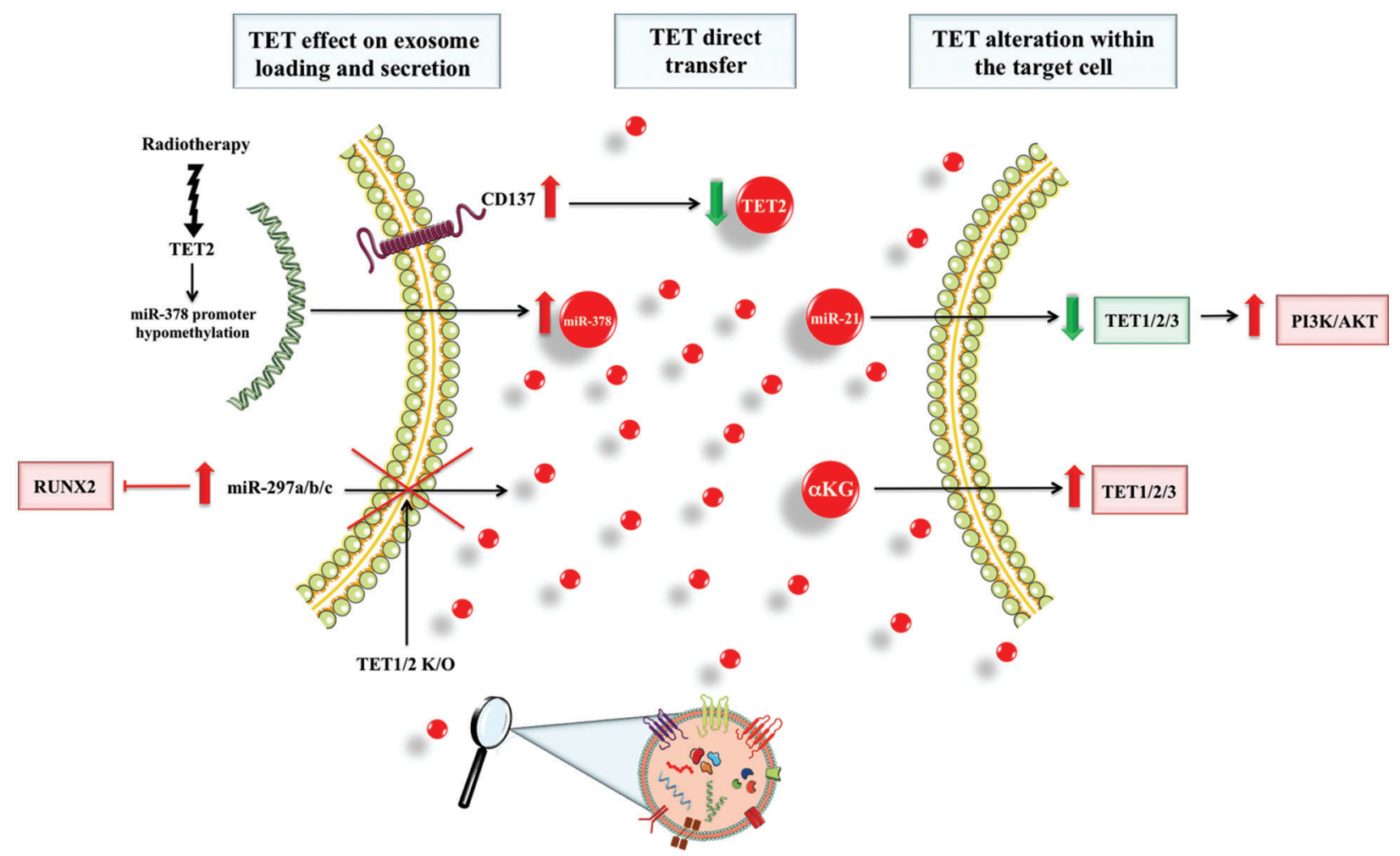

FIGURE 1. Representation of exosome-mediated pathways through which TET1/2/3 are altered in the target cell. Added to this, we also represented the effects that TET1/2 have on exosome loading and secretion within the secreting cell. K/O = knock out.

In the case of hepatocellular carcinoma, exosomes secreted from the malignant clone were shown to have a higher miR-21 content, which can alter the level of TET1/2/3 in the target cell. This, indirectly activates the PI3K/AKT pathway via the TET-mediated alteration in PTENp1 methylation pattern (Cao et al., 2019).

Added to this, certain metabolites that influence the activity of TET in the target cell can be transferred via exosomes. One important example in this direction is represented by the transfer of a-keto-glutarate to macrophages, which leads to the skewing of these cells towards an M2 (anti-inflammatory) phenotype, in a TETmediated manner (Liu et al., 2018). This is important as TET enzymes and, especially TET2, are known to have importance in affecting macrophage polarization and, thus, the inflammatory effects of these cells (Liu et al., 2021).

TET alteration within the secreting cell

Alteration of TET enzymes activity can also affect both exosome loading and secretion. TET1/2 knock-out experiments in bone marrow mesenchymal stem cells showed that exosome secretion is reduced, which further leads to the intracellular accumulation of $\mathrm{miR}-297 \mathrm{a} / \mathrm{b} / \mathrm{c}$. This is important as these reduce the activity of RUNX2, thus inhibiting the role of bone marrow mesenchymal stem cells in bone formation, and further explaining the osteopenic phenotype a TET1/2 knock-out mice model presents (Yang et al., 2018). Added to this, irradiation of tumors was shown to decrease the methylation of miR-378 promoter in a TET2-mediated manner, leading to its overexpression and increased loading into exosomes. In this context, the transfer of miR-378 to NK-cells leads to the decrease of granzyme- $\mathrm{B}$, thus reducing the immune activity of these cells and offering a biological cue to the acquisition of resistance to radiotherapy (Briand et al., 2020).

\section{Perspectives}

In the future it is possible that more studies showing the interdependence between exosomes and TET signaling will be made. Considering the wide range of processes in which TET signaling is a key component of (Tanaka et al., 2020; Oh et al., 2020; Pasca et al., 2021), it is possible that the current studies only scratched the surface of the interplay between TET signaling and exosomes. Thus, it is possible not only that further studies will deepen the understanding of how TET or its alterations can be transferred via exosomes, but also, given its role in immunity, it is possible that these pathways could be targeted in the future in the fields on oncology, autoimmunity and atherosclerosis.

Authors' Contribution: A.J. and S.P. wrote and edited the manuscript during its preparation and approved the final version of the manuscript.

Funding Statement: S.P. was supported by an internal grant of the "Iuliu Haţieganu" University of Medicine and Pharmacy (UMPh), Cluj-Napoca, for sustaining his Ph.D. Research Project.

Conflicts of Interest: The authors declare that they have no conflicts of interest to report regarding the present study.

\section{References}

Aslanyan MG, Kroeze LI, Langemeijer SMC, Koorenhof-Scheele TN, Massop M et al. (2014). Clinical and biological impact of 
TET2 mutations and expression in younger adult AML patients treated within the EORTC/GIMEMA AML-12 clinical trial. Annals of Hematology 93: 1401-1412. DOI 10.1007/s00277-014-2055-7.

Briand J, Garnier D, Nadaradjane A, Clement-Colmou K, Potiron V et al. (2020). Radiotherapy-induced overexpression of exosomal miRNA-378a-3p in cancer cells limits natural killer cells cytotoxicity. Epigenomics 12: 397-408. DOI 10.2217/epi-2019-0193.

Cao LQ, Yang XW, Chen YB, Zhang DW, Jiang XF et al. (2019). Exosomal miR-21 regulates the TETs/PTENp1/PTEN pathway to promote hepatocellular carcinoma growth. Molecular Cancer 18: 148. DOI 10.1186/s12943-0191075-2.

Jurj A, Zanoaga O, Braicu C, Lazar V, Tomuleasa C et al. (2020). A comprehensive picture of extracellular vesicles and their contents. molecular transfer to cancer cells. Cancers (Basel) 12: 298. DOI 10.3390/cancers12020298.

Kohli RM, Zhang Y (2013). TET enzymes, TDG and the dynamics of DNA demethylation. Nature 502: 472-479. DOI 10.1038/ nature12750.

Li B, Zang G, Zhong W, Chen R, Zhang Y et al. (2020). Activation of CD137 signaling promotes neointimal formation by attenuating TET2 and transferrring from endothelial cell-derived exosomes to vascular smooth muscle cells. Biomedicine \& Pharmacotherapy 121: 109593. DOI 10.1016/j.biopha.2019.109593.
Liu S, Yang J, Wu Z (2021). The regulatory role of $\alpha$-ketoglutarate metabolism in macrophages. Mediators of Inflammation 2021: 5577577.

Liu Z, Gan L, Zhang T, Ren Q, Sun C (2018). Melatonin alleviates adipose inflammation through elevating $\alpha$-ketoglutarate and diverting adipose-derived exosomes to macrophages in mice. Journal of Pineal Research 64: e12455. DOI 10.1111/jpi.12455.

Oh YJ, Shin DY, Hwang SM, Kim SM, Im K et al. (2020). Mutation of ten-eleven translocation-2 is associated with increased risk of autoimmune disease in patients with myelodysplastic syndrome. Korean Journal of Internal Medicine 35: 457464. DOI 10.3904/kjim.2018.247.

Pasca S, Jurj A, Constantinescu C, Zdrenghea M, Tomuleasa C (2021). Implications of TET2 in CAR-T cell activity and target response to CAR-T cell therapy: Lessons learned from $\mathrm{T}$ cells. Critical Reviews in Immunology 41: 13-21. DOI 10.1615/CritRevImmunol.2020036946.

Tanaka S, Ise W, Inoue T, Ito A, Ono C et al. (2020). Tet2 and Tet 3 in B cells are required to repress CD86 and prevent autoimmunity. Nature Immunology 21: 950-961. DOI 10.1038/s41590-020-0700-y.

Wu X, Zhang Y (2017). TET-mediated active DNA demethylation: Mechanism, function and beyond. Nature Reviews Genetics 18: 517-534. DOI 10.1038/nrg.2017.33.

Yang R, Yu T, Kou X, Gao X, Chen C et al. (2018). Tet1 and Tet2 maintain mesenchymal stem cell homeostasis via demethylation of the P2rX7 promoter. Nature Communications 9: 2143. DOI 10.1038/s41467-018-04464-6. 